\title{
An early warning indicator for liquidity shortages in the interbank market
}

Article

Accepted Version

Eross, A., Urquhart, A. and Wolfe, S. (2019) An early warning indicator for liquidity shortages in the interbank market. International Journal of Finance \& Economics, 24 (3). pp. 1300-1312. ISSN 1099-1158 doi:

https://doi.org/10.1002/ijfe.1719 Available at https://centaur.reading.ac.uk/81419/

It is advisable to refer to the publisher's version if you intend to cite from the work. See Guidance on citing.

To link to this article DOI: http://dx.doi.org/10.1002/ijfe.1719

Publisher: Wiley-Blackwell

All outputs in CentAUR are protected by Intellectual Property Rights law, including copyright law. Copyright and IPR is retained by the creators or other copyright holders. Terms and conditions for use of this material are defined in the End User Agreement.

\section{www.reading.ac.uk/centaur}

\section{CentAUR}

Central Archive at the University of Reading 
Reading's research outputs online 


\title{
An early warning indicator for liquidity shortages in the interbank market
}

\author{
Andrea Eross: a.eross@hw.ac.uk 1 \\ Andrew Urquhart: a.j.urquhart@soton.ac.uk ${ }^{2}$ \\ Simon Wolfe: ssjw@soton.ac.uk ${ }^{2}$
}

\begin{abstract}
This study investigates an early warning indicator for liquidity shortages in the short-term interbank market. To identify structural breaks and their persistence, an autoregressive two-state regime switching model is presented. The variability in the LIBOR-OIS spread along with thresholds, which delimit four intensities, reveal regime changes consistent with liquidity crashes. The transition between the states is state dependent, and the posterior estimates for the crisis and non-crisis states are estimated using the Gibbs sampler. We forecast our early warning indicator up to December 2011 and show that the estimates are superior to a random walk with drift. Therefore, the model is an effective early warning indicator of an imminent liquidity shortage impacting the interbank market.
\end{abstract}

Keywords: Early warning indicator, Interbank market, Liquidity crises, Regime switching, Bayesian

JEL classification: C11, F37, G01

\section{Introduction}

The financial crisis of 2007-08 is recognised to be the worst crisis since the Great Depression of the 1930s and as a result, liquidity risk in the interbank market has gained increased attention. There is widespread agreement that the two main causes of the 2007-08 credit crisis were inadequate liquidity

\footnotetext{
${ }^{1}$ Accountancy, Economics \& Finance, Heriot-Watt University, Edinburgh

${ }^{2}$ Southampton Business School, University of Southampton
} 
buffers held by banks and lax regulation in the financial system. However, there is a view that the trigger point was a decade earlier, when the US Treasury lowered interest rates to a previously unseen level and kept them there for a prolonged period of time (Economist, 2013). From a monetary policy perspective, the focus was on maintaining low consumer price inflation while ignoring the widespread development of asset price inflation. Consequently, excess liquidity built up in the financial system. This coupled with lack of prudent liquidity measures and prompt reactions led to financial instability.

The Global Financial Crisis was a black swan event, as neither the academic finance community nor finance industry anticipated it. In light of the 2007-08 liquidity crisis, our study investigates whether there was an early warning signal before the crisis erupted. More precisely, the analysis is concerned with revealing whether the short-term interbank spread (US LIBOR-OIS) can predict crises, and consequently what are the implications for liquidity risk management. Financial crises are characterised by depth and have their own accelerating moments. To avoid contraction of market activity, sound liquidity management should be in place and thus in times of financial distress pivotal actions can be taken. Measuring liquidity risk is a prerequisite and consequently early warning indicators should be part of sound liquidity management strategies. Triggers, perceived as risk indicators, are explicit early warning signs for each phase (determined by an arbitrary threshold) in a liquidity crisis.

To address the above issues, a regime switching model is proposed which provides the probability of being in a liquidity crisis state at any given instance. $^{3}$ The model is assessed using the daily US LIBOR-OIS spread for a period of 10 years. Responding to the first signs of financial distress is critical in preserving financial stability. The thresholds set in our models signal liquidity deterioration and trace how liquidity risk intensifies into a financial crisis. More precisely, by using four thresholds of different intensities, the depth of financial crises is established.

The US LIBOR-OIS spread can be used to evaluate the health of banks, as it mirrors the risk linked with lending to other banks. As Alan Greenspan argues, the LIBOR-OIS spread remains a barometer of alarm for bank insolvency, and increased spread levels point to difficulties in the banking in-

\footnotetext{
${ }^{3}$ Such models are successfully used in business cycle models, however they have not as yet been applied in the liquidity risk and financial crises literature.
} 
dustry (Thornton, 2009). Greenspan goes further to claim that variations in the LIBOR-OIS spread reveal changes in risk premiums rather than in liquidity premiums, which in turn mirrors banks' need for liquidity. Contrarily, Hui et al. (2011) argue that the LIBOR-OIS spread mainly reflects funding liquidity risk in the interbank market and is used for measuring funding liquidity conditions. The spread includes counterparty-risk premia which originate from the market's perception of a counterparty's ability to repay an interbank loan. Moreover, the spread contains a significant and time-varying funding liquidity element (McAndrews et al., 2008).

Most empirical studies are linear in nature and are not able to capture non-linearities which are particular to circumstances surrounding financial crises (Baba et al., 2008; Schwarz, 2014; Mistrulli, 2011; Upper, 2011; Gorton and Metrick, 2012). Therefore, our study contributes to the empirical literature in several ways. The literature that investigates regime changes in financial markets is very limited and no theory exists for financial crises. Thus, the present study presents a two-state regime-switching Markov chain model which has a predictive quality and is able to identify several crises in the money market spread. The set thresholds can warn of liquidity risk escalation within the interbank market, suggesting that the early warning indicator presented in this study can contribute to a sound liquidity management strategy. Also, the literature does not discuss models which are able to forecast financial crises ex-ante. Moreover, the literature which investigates financial crises initiated by liquidity risk looks at short time intervals, whereas our study covers the period January 2002 to December 2011 in order to reveal several crises and at the same time assessing their dynamics. The models presented in this paper successfully identify liquidity crashes with various intensities, while our out-of-sample forecasts provide superior estimates compared to the estimates of a random walk model. To the best of our knowledge, there is no such study found in the financial crisis or interbank market literature.

The paper is organised as follows. Section 2 surveys the literature, while the methodology and data are discussed in Section 3. Section 4 contains the empirical analysis while section 5 presents the conclusions.

\section{Literature Review}

Broadly, there are three major strands of empirical literature analysing liquidity crises. One refers to the market microstructure of interbank mar- 
kets, while looking at trading behaviour of high frequency data, such as Hartmann et al. (2001), Daníelsson and Saltoğlu (2003), Frank et al. (2008) and Baba et al. (2008). Another focusses on measuring and assessing liquidity risk, such as Brunnermeier (2009), Schwarz (2014), Brunnermeier and Oehmke (2012), Gorton and Metrick (2012), Mistrulli (2011), Zhou and He (2012), Min and Hwang (2012) and Carpenter et al. (2016), while a limited number of studies adopt regime change and Markov models, such as Dahlquist and Gray (2000), Ang and Timmermann (2011) and Guo et al. (2011). The literature investigating the financial crisis of 2007-08 focuses primarily on the analysis of liquidity and credit risk indicators, and decomposition of spreads into credit and liquidity components, revealing which component had a larger influence on widening of the short-term interest rate spreads. Yet, for the period that follows the financial crisis of 2007-08, the majority of studies focus on decomposing spreads into risk components prevalent in bringing down institutions and financial markets.

The early warning literature which focuses on predicting currency and banking crises before the year of 2000 employs a leading indicator approach (Kaminsky et al., 1998; Kaminsky and Reinhart, 1999; Goldstein et al., 2000), whereas the post millennium literature uses predominantly a logit/probit based estimation. The identified crises in these studies are matched against World Bank and IMF bank crisis databases (Caprio et al., 2012; Laeven and Valencia, 2012). Some studies correct for post-crisis bias by proposing discrete-dependent-variable methods with the aim of reducing false crisis warnings as well as revealing ignored crisis periods; such are Demirgüç-Kunt and Detragiache (1998) who drop crisis and post-crisis observations (besides, the literature follows the method of dropping significant observations), while Bussiere and Fratzscher (2006) use a three-state multinomial approach on restricted time intervals to assess the power of economic fundamentals in predicting pre-crisis, crisis and end-of-crisis periods.

Bauer et al. (2007) study the forecasting power of past early warning systems (EWS) considering the high occurrences of Type I and Type II errors. Considering that causes and effects are exclusive for each type of crisis, the predictive power of EWS's can be enhanced if debt-currency crises (namely "twin crises") are classified as a separate crisis category. Barrell et al. (2010) use capital adequacy and liquidity ratios, house prices along with standard indicators used in previous studies (Demirgüç-Kunt and Detragiache, 2005; Berg et al., 2005) to detect banking crises and argue that banks with high capital adequacy and liquidity ratios reduce crisis occurrences, while increased 
house prices contribute to sub-prime episodes. Using the data of Kaminsky and Reinhart (1999) and Demirgüç-Kunt and Detragiache (2005), Davis and Karim (2008) assess the performance of EWSs for banking crises and argue that multivariate logit models are better for designing global EWSs, whereas signal extraction approaches work better for country-specific EWSs. Lang and Schmidt (2016) review leading indicators of banking crises by combining an event study approach with a fan chart and find that accounting for the interaction between broad liquidity ratio and demand deposits improves out of sample forecasts.

An issue with studies based on indicator variable approach is that the binary signal, which crosses an arbitrarily chosen critical threshold, greatly influences the documentation of false crisis occurrences. On the other hand, logit models lack significant components which have the power of describing the severity, persistence and subtleties of time-varying parameter estimates. Moreover, most of the above studies argue that crises are self-fulfilling, however none attempt to identify the endogenous factors that contribute to the self-fulfilling process.

\section{Methodology}

Our model builds on an approach implemented by Martínez-Beneito et al. (2008) who develop a two-state regime-switching Markov model to detect influenza epidemics at the moment of their onset. Our model explores the probability of a financial liquidity crisis developing in the short-term interbank money market. The structure of the model allows for discrete shifts in the mean and for autoregressive dynamics to better illustrate liquidity crashes. In this way, the model is representative of a real world set-up which describes economic and financial time series in turbulent times. As opposed to Martínez-Beneito et al. (2008) who use first differences of the series, the analysis presented in this paper uses the raw data (as stationarity is not a requirement for Bayesian empirical analysis) and thus it maintains the unique characteristics of the short-term interbank spread.

\subsection{Data}

The data used in this analysis is the daily closing US LIBOR-OIS spread obtained from Bloomberg. The time series contains 2609 observations spanning the interval 1st of January 2002 to 30th of December 2011. 
The London Interbank Offer Rate (LIBOR) is used as a reference rate in financial contracts all over the world and is the rate at which banks and institutions of similar size agree to lend to each other. The rate is paid on unsecured interbank loans of various short-term maturities. Fundamentally, a bank with surplus cash credits a bank in need for an agreed time period. Increases/decreases in LIBOR rates are caused by banks calling for greater/lesser compensation in case of default risk on their loans. The Overnight Interest Swap (OIS) rate is the rate of the derivative contract on the federal funds rate, and in usual market circumstances is generally several basis points below the LIBOR rate. In the OIS contract, the interest rate swap's floating leg, which is the federal funds rate, is exchanged for a fixed interest rate. The cash-flow consists of the difference between the two rates exchanged at maturity, and therefore the contract is riskless, as there is no principal involved in the exchange. The present value of the floating rate is calculated generally by taking the geometric average over the length of the contract. The federal funds rate is seen as a good indicator of the health of the short term interbank markets, since it bears no risk compared to traditional interest rate spreads. Moreover, in turbulent market conditions, the LIBOR-OIS spread is a good indicator of risk premiums as a result of credit risk and funding liquidity risk.

During the financial crisis of 2007-08, the spread varied persistently around record high levels until the announcement of Lehman Brothers' collapse. Since then, for the period of investigation, the spread level continued to fluctuate, however it did not stabilise relative to its pre-2007 level. Our results reveal that the US LIBOR-OIS spread is a good indicator of financial instability. For the interval January 2010 to January 2012, the European Central Bank bailed out Ireland once and Greece twice. Interestingly, all these three events were reflected in the US LIBOR-OIS spread movements over that time period.

\subsection{Model specification}

Constructing a regime switching model with state dependent probabilities results in a parsimonious model with very few assumptions. The structure is characterised by a hidden two-state Markov chain of order 1, meaning that the future state (or regime) will only depend on the present state. Essentially, the data is modelled as partly observed as the states are latent and the LIBOR-OIS spread and the final output is observable, which is conditional on the state variable and time. 
The following equation describes the LIBOR-OIS spread and also summarizes the assumed data generating process. $Y_{t}$ corresponds to a stochastic process and the model contains an autoregressive component, as follows:

$$
Y_{t}=\rho Y_{t-1} I_{t}+\epsilon_{t}
$$

where $t=1,2, \ldots, T$ represents the number of observations in the series, in this case 2609, and $\rho$ is the autoregressive coefficient. ${ }^{4}$ Furthermore, it is assumed that:

$$
Y_{t} \sim N\left(\mu_{t}, \tau_{t}\right)
$$

where the time and state dependent $\tau$ is the precision parameter and corresponds to $\tau=1 / \sigma^{2}$.

The $I_{t}$ is the binary (dummy) indicator which can take the following values:

$$
I_{t}= \begin{cases}1, & \text { if there is crisis in the short term money market; } \\ 0, & \text { if there is no crisis in the short term money market. }\end{cases}
$$

Furthermore, it is also assumed that the LIBOR-OIS spread is directly observable and its dynamics change in line with the value of the crisis variable, that is with $I_{t}$. Fundamentally, the change from one state to another will be a function of the LIBOR-OIS spread and its lag, as indicators. The state variable $\left(I_{t}\right)$ is latent and is supposed to progress corresponding to a first-order Markov chain with transition probabilities controlled by a set of covariance-stationary exogenous variables (Gelman et al., 2013). ${ }^{5}$

There are two error terms in the system of equations, one corresponding to the non-crisis and the other to the crisis equation. The error terms must satisfy the following:

$$
\epsilon_{\mathbf{0} t} \sim^{i i d} N\left(0, \sigma_{0, t}^{2}\right)
$$

\footnotetext{
${ }^{4}$ The rationale of using an autoregressive model for estimation comes from the work of Akaike (1969).

${ }^{5}$ The exogenous variable can take the form of an external shock such as a new macroeconomic policy rule or an announcement made by a leading investment bank, for example.
} 


$$
\epsilon_{\mathbf{1} t} \sim^{i i d} N\left(0, \sigma_{1, t}^{2}-\sigma_{0, t}^{2}\right)
$$

with

$$
\sigma_{1, t}^{2}-\sigma_{0, t}^{2}>0
$$

This last assumption is due to the fact of random variations in the data (Martínez-Beneito et al., 2008), and not due to the effect of a financial crisis, for example. Also, it is assumed that the two error terms are not correlated.

The model presented in Equation 1 makes posterior inference about the 'true' crisis time $\theta$. Theta is paired with a set of explanatory variables $X_{1}, X_{2}, \ldots X_{T}$ (in this case $\mu, \tau, \lambda_{1}, \lambda_{2}$ and $I$ ) with the aim of stating the link function and the ultimate structure of the model (Kim and Nelson, 1999). The model estimates the posterior parameter values that enclose both priors for all the parameters of the model and observed data information. ${ }^{6}$

The system consists of the state space, the combined parameter vector, the regime matrix describing the probabilities of individual transitions and an initial state. The aim is to infer the posterior distribution given the fluctuation in the LIBOR-OIS time series. It is assumed that the initial state of the system is a non-crisis one. As $t \rightarrow \infty$, the unconditional distribution of $X^{t+1}$ converges to an exclusive stationary distribution. The marginal distributions, parameters and two regimes (crisis and normal times) are estimated using the Gibbs sampler, which is a randomised system where iterations are being run and are continuously revised. ${ }^{7}$ The rationale of adopting the Gibbs sampler is the appealing fact that convergence is achieved accurately and quickly, regardless of the dimension of the coefficient vector which is estimated. Following the specification of an arbitrary set of starting values - which is not compulsory - and running a fairly large number of iterations, marginal distributions are estimated by convergence to the true joint density value.

\footnotetext{
${ }^{6}$ The interested reader can find information on how the prior distributions of the variables, likelihood function, sampling, posterior distribution and state probabilities are calculated in Appendix A and Appendix B.

${ }^{7}$ The Gibbs sampler introduced by Geman and Geman (1984), is an updating process of an iteration with values that have been drawn from a system. The individual full conditional distribution is governed solely by some "neighbourhood subset of variables".
} 
We run four models (see Table 1) to determine which one provides with superior estimates. The ultimate aim is to reveal the dynamics of the US LIBOR-OIS spread and show if it can act as an early warning indicator inside a liquidity management strategy to predict and mitigate liquidity crashes. The equation of Model 1 does not contain a constant, and for all models the regime changes occur in the mean. The autoregressive coefficient $\rho$ and the constant is allowed to vary within set intervals, as shown below. In Model 4, changes in the constant are attributed to changes in the variance.

Table 1: Model characteristics for Model 1, 2, 3 and 4.

\begin{tabular}{lcccc}
\hline Model & M1 & M2 & M3 & M4 \\
\hline \hline constant in the equation & no & yes & yes & yes \\
regime switch & mean & mean & mean & mean \\
variations in the constant & no & no & no & yes \\
$\rho$ & {$[0,1.2]$} & {$[0,1.2]$} & {$[0,1.5]$} & {$[0,1.5]$} \\
constant & - & {$[-40,40]$} & {$[-400,400]$} & {$[-400,400]$} \\
\hline
\end{tabular}

\subsection{Model validation and selection}

In a Bayesian setting, one is expected to centre a comparative scale on the posterior distribution of the deviance, or to use Bayes factors, namely the Bayesian Information Criteria (BIC).

The Deviance Information Criteria (DIC) (Spiegelhalter et al., 2002) is a test of absolute fit, and is consequently used to validate and select the best model out of the four presented later.

Essentially, the DIC is a generalisation of the AIC and is calculated by the following expression:

$$
D I C(m)=2 \overline{D\left(\theta_{m}, m\right)}-D\left(\overline{\theta_{m}}, m\right)=D\left(\overline{\theta_{m}}, m\right)+2 p_{m}
$$

where $D\left(\theta_{m}, m\right)$ is the deviance measure (as a function of $\theta$ ) given by:

$$
D\left(\theta_{m}\right)=-2 \log p(Y \mid \theta)
$$


$\overline{\theta_{m}}$ is the posterior mean of the coefficients of the model $m$ and $p$ represents the number of efficient parameters in the model, given by:

$$
p_{m}=\overline{D\left(\theta_{m}, m\right)}-D\left(\overline{\theta_{m}}, m\right)
$$

The use of the DIC is fairly simple. For the reason that all four models are autoregressive, one does not have to perform stepwise elimination of variables from the regression equations. After running 10,000 iterations, estimated posterior distributions of parameters are reported and the DIC is obtained. A low DIC is preferred over a high value.

\subsection{Forecasting the short-term interbank spread}

Forecasting crises in the LIBOR-OIS spread is undeniably valuable to financial institutions and policy makers; it enables to identify difficulties and risks in the money market and make adjustments to avoid or mitigate challenges and threats caused by amplified volatility and persistent uncertainty, which can destabilise the financial system and eventually cripple the wider economy. The aim is to show that the US LIBOR-OIS spread possesses the features of an early warning indicator.

Let $\boldsymbol{Y}$ represent the LIBOR-OIS time series of $T$ sample realisations of a random process for the period $t=1,2, \ldots, 1399 ; \boldsymbol{Y}=\left[y_{1} y_{2} \ldots y_{1399}\right]^{\top}$. Therefore, the first 1399 observations of the LIBOR-OIS spread series are

used to forecast the subsequent period, which includes the financial crisis of 2007-08 and the Eurozone crisis that followed. The last observation of $t$ (on the timescale $1,2, \ldots, 1399$ ) corresponds to 11th of May 2007, when there were no apparent signs (such as official announcements) of a liquidity crash in the short-term interbank market in the US nor in the Eurozone market. Now let $\boldsymbol{Y}^{\boldsymbol{p}}$ represent a vector of future observations of the LIBOR-OIS time series; $\boldsymbol{Y}^{\boldsymbol{p}}=\left[y_{T+1} y_{T+2} \ldots y_{T+q}\right]^{\top}$.

$$
p\left(\boldsymbol{Y}^{\boldsymbol{p}} \mid \boldsymbol{Y}\right)=\int_{\Theta} p\left(\boldsymbol{Y}^{\boldsymbol{p}}, \theta \mid \boldsymbol{Y}\right) d \boldsymbol{\theta}=\int_{\Theta} p\left(\boldsymbol{Y}^{\boldsymbol{p}} \mid \theta, \boldsymbol{Y}\right) p(\boldsymbol{\theta} \mid \boldsymbol{Y}) d \boldsymbol{\theta}
$$

Essentially, the predictive probability distribution of $\boldsymbol{Y}^{\boldsymbol{p}}$ is conditional on the 1399 observations. The model forecasts the next 1210 observations using the Gibbs sampler.

The model estimates are compared to estimates of the random walk model with drift which is a special case of the $\mathrm{AR}(1)$ model. The main question we 
ask: does our model have a superior predictive power in forecasting financial crises compared to that of a random walk model?

The random walk model is defined as follows:

$$
Y_{t}=\alpha+\beta Y_{t-1}+u_{t}
$$

where $Y_{t}$ is a non-stationary process with the coefficient $\beta=1, \alpha$ is the drift component and $u_{t}$ is a white noise process.

To assess the accuracy of the estimates, the estimated standard errors of the two models are compared. In Bayesian estimation, the standard deviation of the mean and the Monte Carlo standard errors are used to gauge the precision of a model. These two criteria depend on the number of simulations executed as well on the number of observations used. By measuring the variance of the asymptotic distribution within a valid confidence interval, one can actually estimate the Monte Carlo standard error, which in turn evaluates the precision of the point estimate (Flegal et al., 2008).

\section{Results}

Figure 1 shows the behaviour of the LIBOR-OIS spread over the period January 2002 - December 2011. As opposed to simple Markov chains where the states are observable, in this analysis the crisis and tranquil states are latent. By implementing a two-state Markov regime switching method, the models presented below segment the LIBOR-OIS spread into crisis and non-crisis intervals. To make our results more informative, we use thresholds to reveal four intensities in the LIBOR-OIS spread fluctuations. Let $Y=\left\{Y_{t} ; \mathrm{t}=1, \ldots, 2609\right\}$ represent the time series running from 1st January 2002 to 30th December 2011. In the next subsections four models are presented (see Table 1 for a summary of the models). As a prerequisite for latent Markov chain models, the starting state must be determined from the outset. Therefore, for all four cases, it is assumed that at $t=1$ the system is in a non-crisis state. To forecast the crisis and non-crisis periods, a binary state indicator variable $I_{t}$ is included in the regression equations. For all the models, the first 1,000 iterations (corresponding to the burn-in period) are discarded, then a further 10,000 samples are run. The resulting projected densities are plotted and visually inspected. Interestingly, after only 2000 samples, the desired marginal distributions converged to their expected stationary values. 


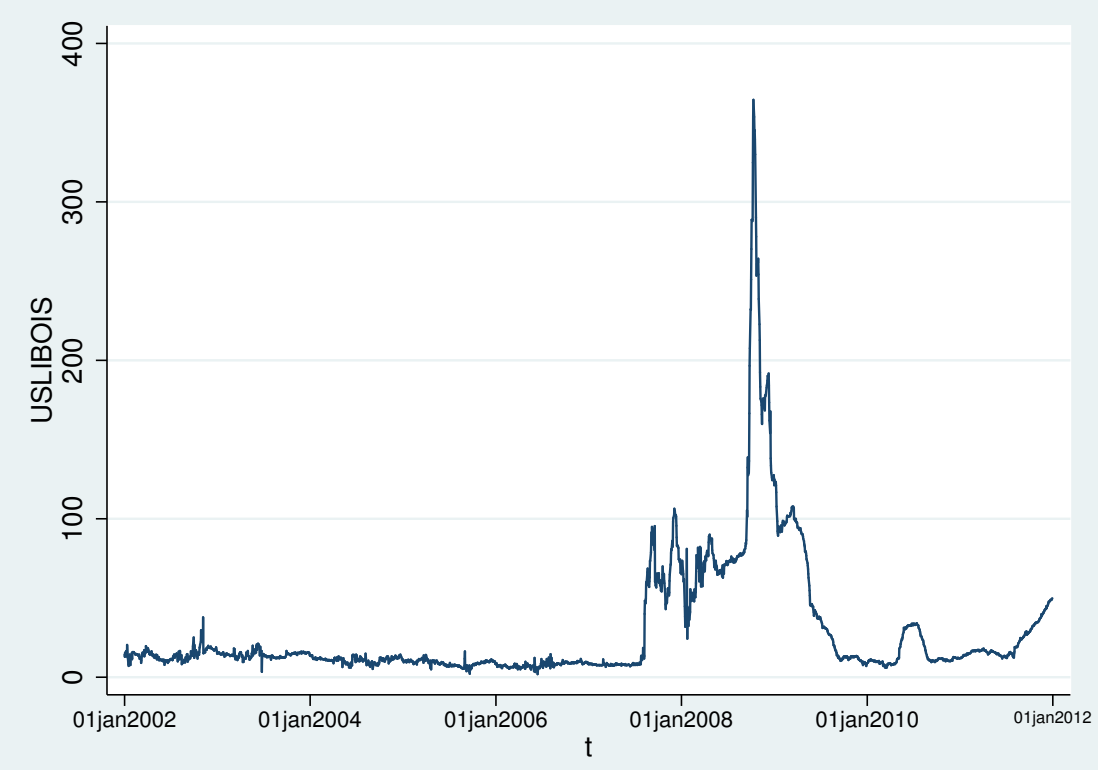

Figure 1: Behaviour of the daily US LIBOR-OIS spread for the period 1st January 2002 to 30th December 2011.

\subsection{Model 1 (M1)}

The model below assumes that the regime switch occurs only in the mean. As explained earlier, the innovations follow a Gaussian normal distribution with zero mean and variance $\sigma_{0, t}^{2}$ and $\sigma_{1, t}^{2}$, respectively. The explanatory variable is the observed autoregressive component which is state and time dependent. There is no constant included in the equation. The state parameter follows a categorical distribution and can take the values 0 and 1. The transition parameters follow the Beta distribution, whereas the remaining parameters follow a univariate probability distribution. For values $t=2, \ldots, 2609$, the expected mean is determined as follows:

$$
E\left(Y_{t}\right)=\rho Y_{t-1} I_{t, 2}+\epsilon_{t}
$$

where $\epsilon_{t}$ is a white noise error. Considering that the process in a crisis period is assumed explosive, the autoregressive coefficient $\rho$ is arbitrarily set to take any value between $[0,1.2] . \tau$ is the precision parameter satisfying $\tau=\frac{1}{\sigma^{2}}$; thus, $\tau$ is the inverse of the variance. The minimum value of the spread is at 1.91 and the maximum is at 364.43 (see Figure 1). The values of the 
hyper-parameters $a$ and $b$ in Equations B.6 to B.11 (see Appendix 2) are fixed at 0 and 370 respectively.

\subsection{Model 2 (M2)}

A constant (intercept) is included in the equation; this ensures that the residuals are mean reverting. The interval of the autocorrelation coefficient $\rho$ is limited to vary between $[0,1.2]$ and the constant is allowed to vary in the interval arbitrarily set at $[-40,40]$.

$$
E\left(Y_{t}\right)=\alpha+\rho Y_{t-1} I_{t, 2}+\epsilon_{t}
$$

\subsection{Model 3 (M3)}

The difference between Model 2 and Model 3 is that the autoregressive coefficient and the constant are allowed to vary in the interval $[0,1.5]$ and $[-400,400]$ respectively. It is expected that larger intervals for the autoregressive coefficient and constant would better support the model in identifying crises more accurately over the 10 year period of analysis.

\subsection{Model 4 (M4)}

In this model, variations in the level are attributed to changes in the variance, while the mean changes regimes, as follows:

$$
E\left(Y_{t}\right)=\alpha \tau_{t}+\rho Y_{t-1} I_{t, 2}+\epsilon_{t}
$$

Both the time dependent constant and noise terms for the two regimes follow a univariate distribution. Similarly to Model 3, the autoregressive coefficient and the constant can vary in the interval $[0,1.5]$ and $[-400,400]$ respectively.

Table 2 presents the posterior parameter estimates and their $95 \%$ credible interval for the four models described above. A single chain of simulations with 10,000 iterations was run, as this proved sufficient for the posterior parameters to converge to their true value. Figure 2 graphs the "crisis" parameter with the two periods for the four distinct models. The simplest model, represented by Model 1, identifies eight crises. As it can be seen in Figure 4, where Model 1's crisis output is mapped, the regime estimates are close to or equal to 1 - in the case of a tranquil phase -, and they are close to or equal to 2 - indicating a crisis phase. All values equal to or above 1.5 are 
considered to correspond to a crisis state. It appears that different types of crises are mirrored in liquidity crises observed in the interbank market. Such is the Tech Market Crash of 2000 and the Stock market crash of 2002. The high unemployment rate perceived in the US in 2002-2003 and the Iraq War also affected the money market. The Global Financial Crisis was instigated by liquidity crisis, whereas the European debt crisis was a direct consequence of the credit crunch (as acknowledged by academics and the industry).

If a constant is added to the equation as is for Model 2 and 3 (see Figure 2 ), there is a short period from end of 2003 to the beginning of 2004 where the models perceive some disturbance in the money market, however it did not materialise in a crisis. If changes in the level are allowed to be affected by the variance while the mean is changing regimes, such as in Model 4, for the period ranging from 1st January 2002 to 30th December 2011, 12 crises are identified. Unquestionably, most of these are false crisis occurrences pointing to an over-reacting model.
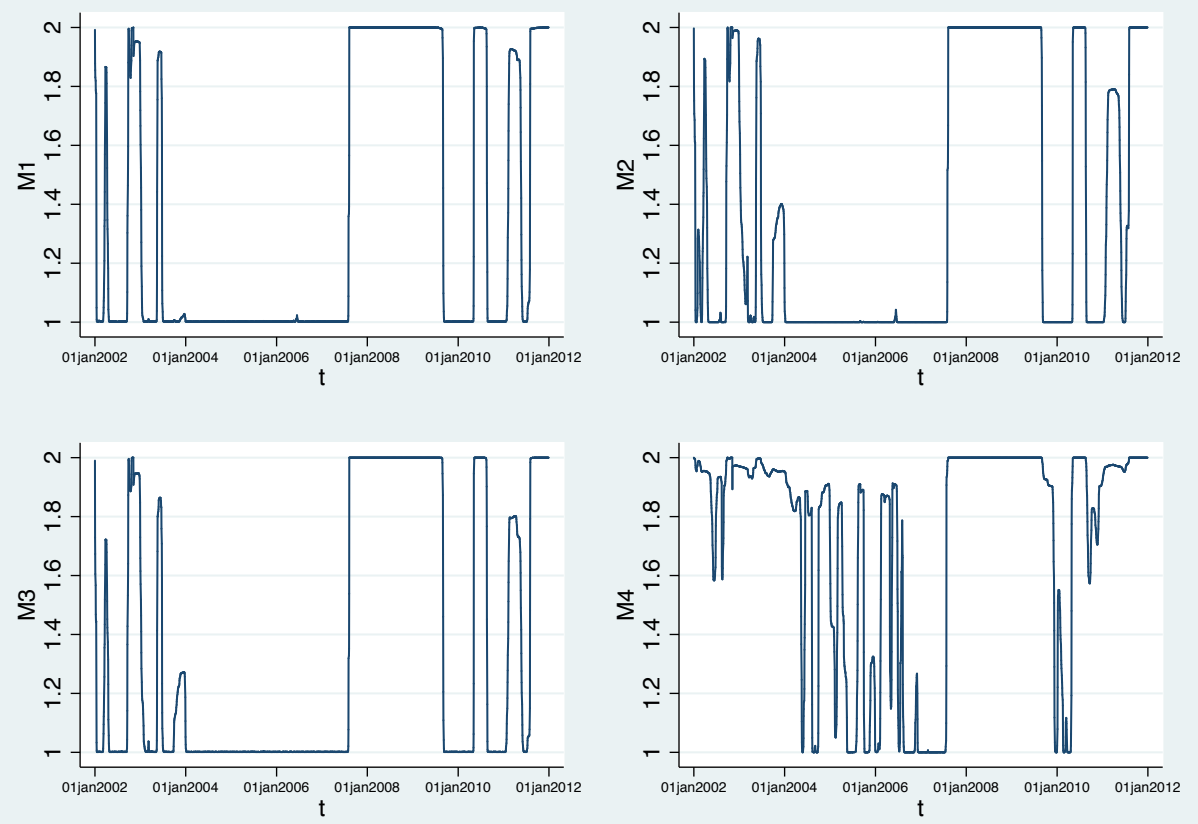

Figure 2: State prediction for Model 1, Model 2, Model 3 and Model 4 versus time for the period 1st January 2002 to 30th December 2011.

The estimated parameters efficiently characterise the dynamics of the two 
Table 2: Estimated parameter values, regime change probabilities and DIC values.

\begin{tabular}{|c|c|c|c|c|}
\hline Parameters & M1 & M2 & M3 & M4 \\
\hline$\overline{\theta_{i n f}}$ & $\overline{1.69}$ & 1.665 & $\overline{1.923}$ & 0.802 \\
\hline $95 \%$ interv. & {$[0.1316,2.707]$} & {$[0.1281,2.783]$} & {$[0.1382,2.98]$} & {$[0.05109,2.223]$} \\
\hline$\theta_{\text {med } 1}$ & 4.015 & 3.897 & 4.323 & 1.828 \\
\hline $95 \%$ interv. & {$[2.694,7.192]$} & {$[2.454,7.014]$} & {$[2.622,7.218]$} & {$[0.9262,3.621]$} \\
\hline$\theta_{\text {med } 2}$ & 6.462 & 6.382 & 7.046 & 2.985 \\
\hline $95 \%$ interv. & {$[3.378,8.562]$} & {$[3.342,8.534]$} & {$[3.427,8.61]$} & {$[1.407,4.685]$} \\
\hline$\theta_{\text {sup }}$ & 75.79 & 72.43 & 73.25 & 63.08 \\
\hline $95 \%$ interv. & {$[8.674,330.6]$} & {$[8.594,325.3]$} & {$[8.6,327.1]$} & {$[3.924,320.5]$} \\
\hline$\rho$ & 0.4291 & 0.9049 & 0.9048 & 0.9959 \\
\hline$P_{0,0}$ & 0.9948 .5 & 0.9948 & 0.9951 & 0.9821 \\
\hline$P_{0,1}$ & 0.00515 & 0.005186 & 0.004942 & 0.0179 \\
\hline$P_{1,0}$ & 0.00838 & 0.008771 & 0.008257 & 0.006261 \\
\hline$P_{1,1}$ & 0.9916 & 0.9912 & 0.9917 & 0.9937 \\
\hline DIC & 1798 & 1716 & 1767 & 1881 \\
\hline
\end{tabular}

states, and for Models 1, 2 and 3 the estimated intervals correspond accurately to the dating of identified crisis and non-crisis periods. Liquidity crises identified by the models centred on the dynamics of the LIBOR-OIS spread perfectly match crises observed from 2002 to the beginning of 2012 (see Figure 4). This suggests that the LIBOR-OIS spread not only evaluates the health of banks and financial stability, but that of the entire economy.

By defining the transition probabilities and thresholds $\theta_{\text {inf }}, \theta_{\text {mid } 1}, \theta_{\text {mid } 2}$ and $\theta_{\text {sup }}$, the model also explains the likely mean values of spreads being in an inferior-, lower-mid-, upper-mid- and superior phase (see Figure 3). The thresholds are not fixed, and therefore the models define crises in the 


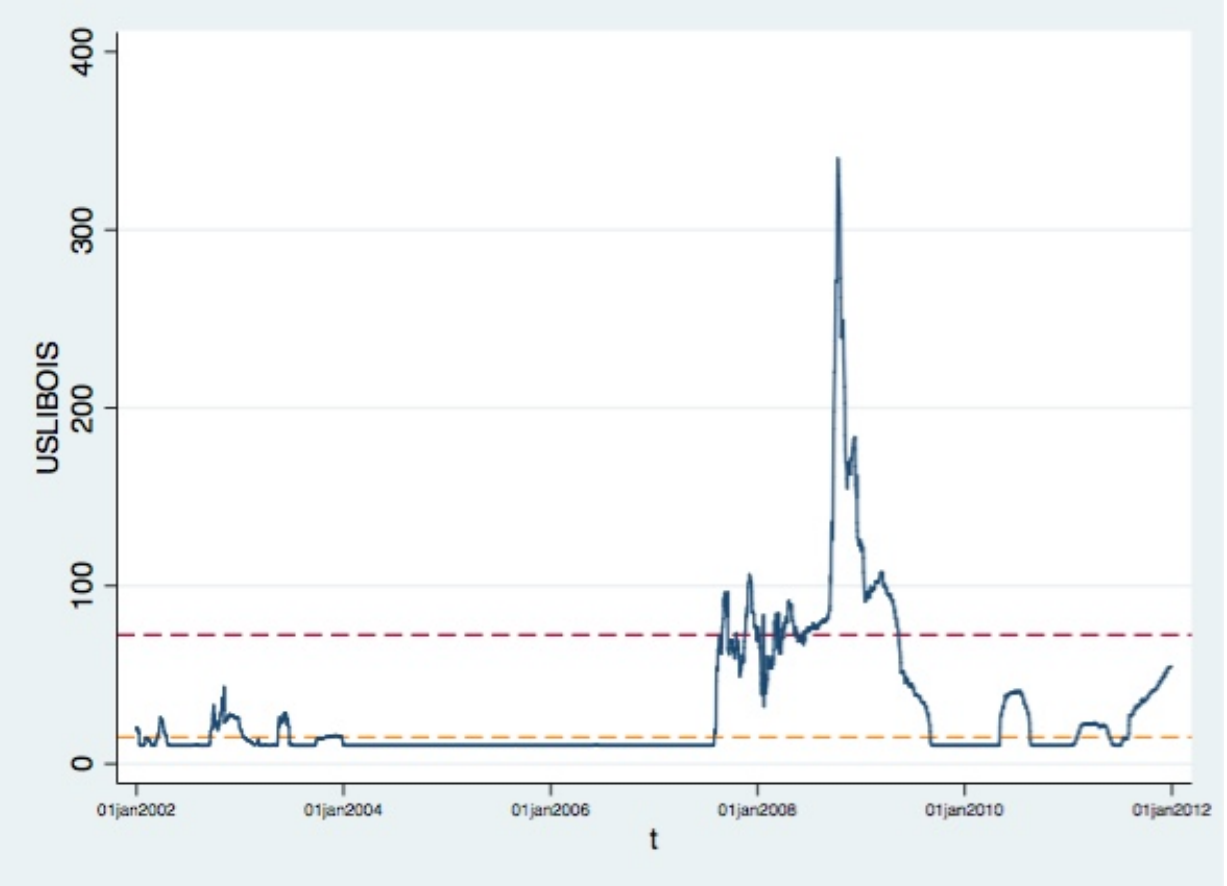

Figure 3: The estimated mean for the US LIBOR-OIS spread with the superior crisis threshold in red $\left(\theta_{\text {sup }}\right)$ at value 72.43 and the upper medium crisis threshold in orange $\left(\theta_{\text {med2 }}\right)$ at value 6.382 denoting the depth of crises for Model 2.

LIBOR-OIS spread with two different intensities; for example, as seen in Table 2 , the $95 \%$ credible interval for $\theta_{\text {mid } 2}$ corresponds to a low intensity crisis, whereas $\theta_{\text {sup }}$ corresponds to a high intensity crisis period and liquidity risk contagion within the financial market is likely to occur, eventually leading to financial crisis. With a $95 \%$ confidence level, the posterior mean representing crisis level $\theta_{\text {sup }}$ for Model 2, has a value of 72.43 and falls within the interval $[8.594,325.3]$. Thus, observations that take values within this interval are assumed to correspond to a crisis period. On the contrary, values in the interval $\theta_{\text {inf }}=[0.1281,2.783]$ correspond to tranquil periods. When the values of the LIBOR-OIS spread are around 3.897 (which is the mean value for $\theta_{\text {mid } 1}$ ), precautionary measures could be taken by institutions, for example, to mitigate liquidity risk.

The autoregressive coefficient has near unity value for Models 2,3 and 4 . The financial crisis of 2007 had an impact on the parameter estimates since it translates into explosive jumps in the spread, and for the period August 2007 
to June 2009 the estimated mean values are continuously in the crisis state. We argue that explosive spread rates are associated with crisis time, and low spread values with non-crisis periods. There are clear breaks between the crisis and non-crisis phases as seen in Figure 2 and these correspond to regime switches. For all four models, the transition parameter estimates show that there is a high probability to be in a non-crisis state if the previous state was also a non-crisis one (see the $P_{0,0}$ values, for example in Table 2), exhibiting $99 \%$ probability for the first three models, and $98 \%$ probability for the fourth model. Similarly, a crisis state is more likely to be followed by a crisis state, as picked up by the transition estimates $P_{1,1}$, for example with $99 \%$ probability for all four models. This is consistent with the volatility clustering phenomenon seen in time-series during turbulent times. The transition estimates for $P_{0,1}$ and $P_{1,0}$ indicate the probability of regimes switching from tranquil to crisis and from crisis to tranquil periods, correspondingly. As expected, these take very low values (less than $0.1 \%$ ), conveying that during tranquil periods, the probability of an intensified liquidity crisis emerging within the money market is extremely low; similarly, if the money market is in the mids of financial distress, the probability of swift calming is very low. It takes rather a prolonged period of time for markets to stabilise after a financial crisis.

The DIC is lowest for Model 2 with a value of 1716 . Model 1, 3 and 4 had values of 1798, 1767 and 1881, respectively. Thus, it can be concluded that Model 2 performed best. Visual inspection of Figure 2 corroborates the findings and shows that Model 2 identifies the crises periods more accurately than the other three models.

\subsection{Out-of-sample forecasting to detect liquidity crises}

We use Model 2 to predict the mean values for the period May 2007 - December 2011. To ensure convergence when estimating the forecasted values of the US LIBOR-OIS spread, the first 1000 iterations are discarded and further 10,000 number of iterations are run; the decision on how many iterations are needed is based on the Gelman-Rubin diagnostics (Gelman and Rubin, 1992). The standard deviation of the mean (also known as standard errors) and Monte Carlo errors were obtained.

Figure 5 maps our Forecasting model predictions, the random walk with drift estimates and the LIBOR-OIS time series and shows that the forecasts are closer to the values of the LIBOR-OIS spread than the random walk 


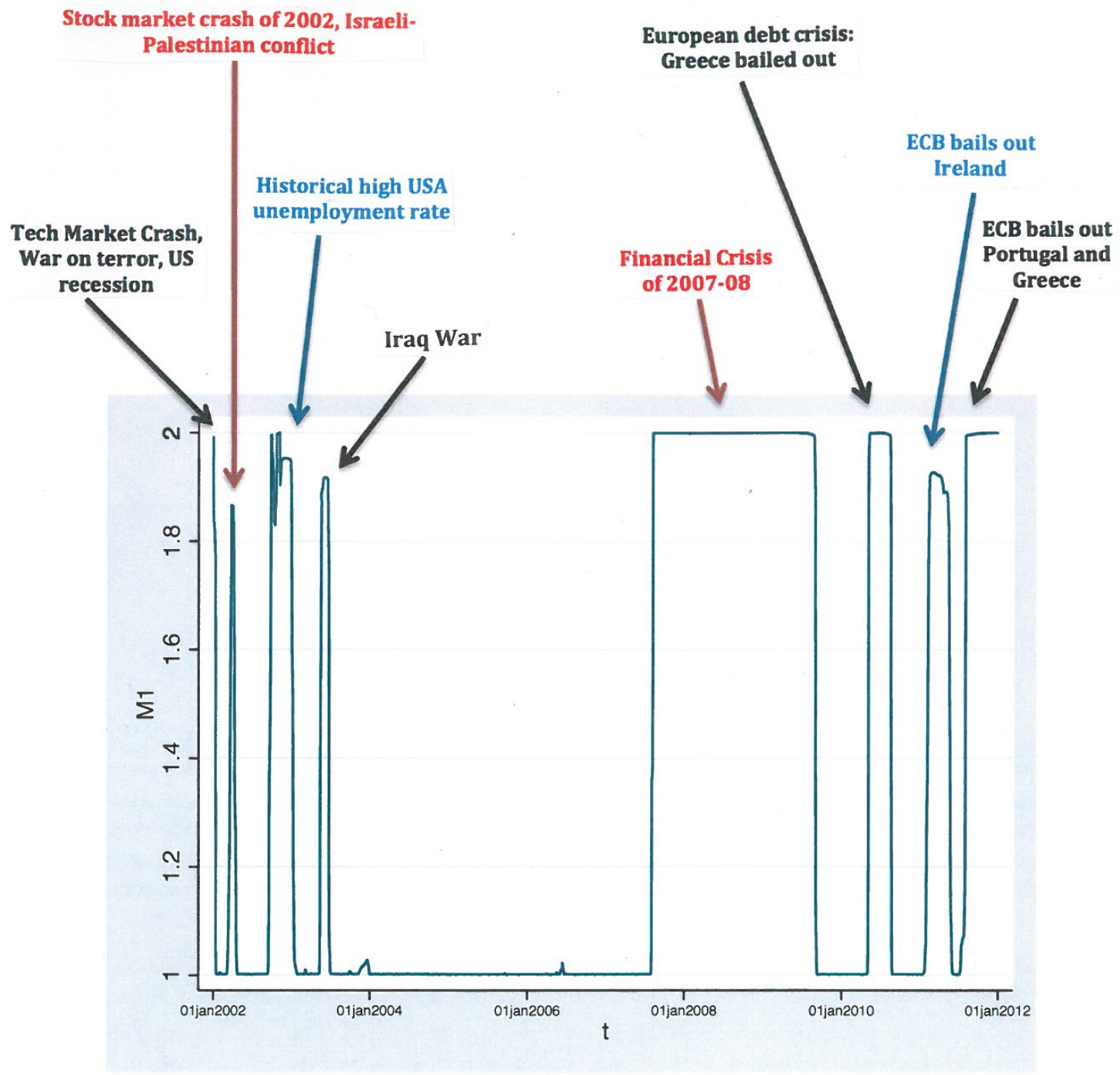

Figure 4: Timeline with the crises identified by Model 1. 


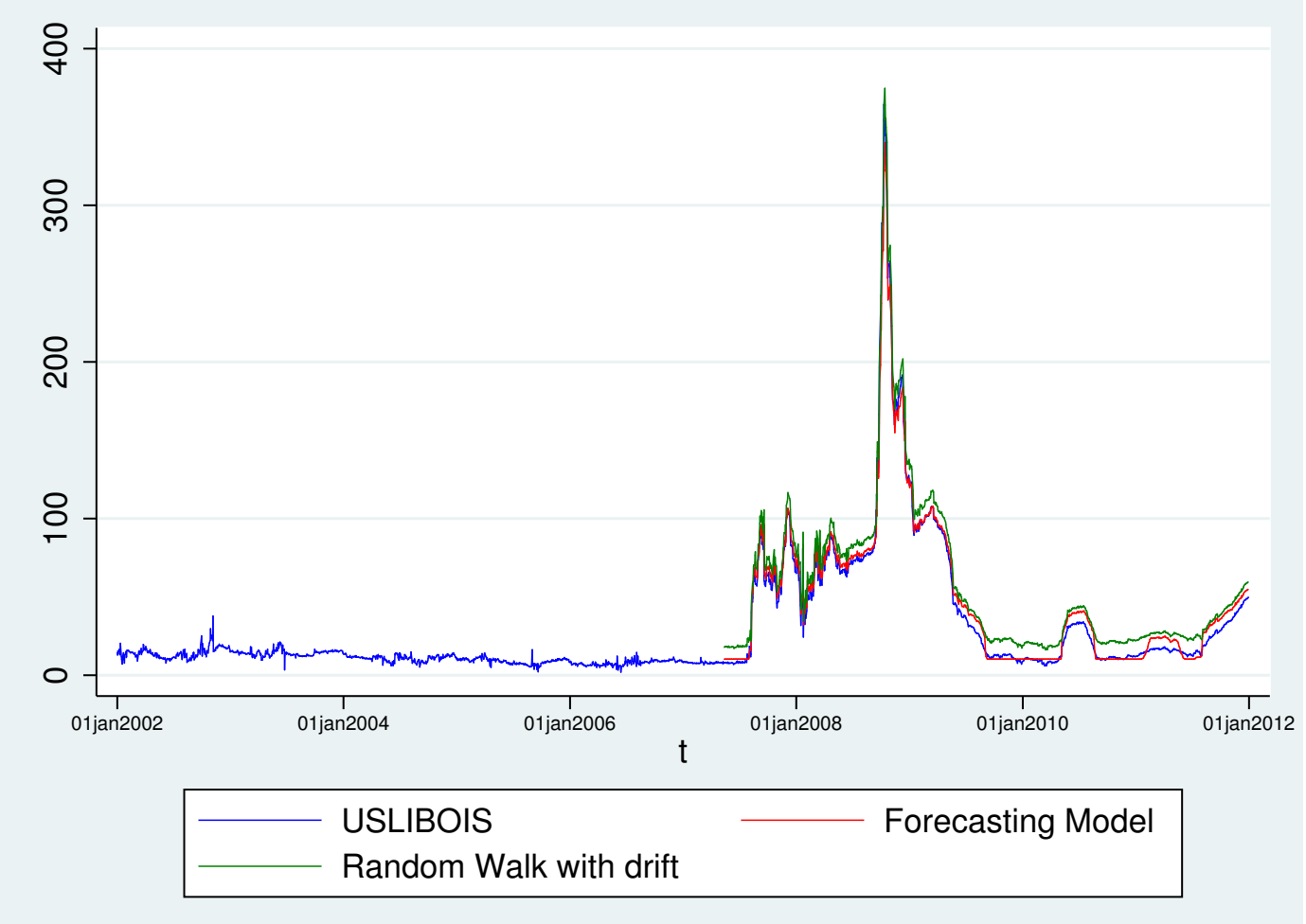

Figure 5: Expected mean values of the Forecasting and Random Walk with drift models versus the LIBOR-OIS spread. 
model estimates. To determine the reliability of predicted values, the standard errors are examined. At 95\% confidence interval, the posterior standard error of the mean estimate for our model is 0.011 , whereas the point estimate of the standard error for the random walk model is 0.0356. Based on both the mean estimates and standard error values, we argue that our forecasting model performs better than the random walk model, as it improves on the forecast that the US LIBOR-OIS spread will be the same tomorrow as it is today.

\section{Conclusions}

This study traces liquidity crises in the daily US LIBOR-OIS spread employing an autoregressive Markov regime switching model and determines the length of such turbulent periods. The above regime switching model has only a mean specification and assesses the period of 1st January 2002 to 30th December 2011. Gibbs sampling, the algorithm behind the Markov chain Monte Carlo method, is used to estimate posterior mean probabilities of regime changes. The posterior distribution is the foundation for statistical interpretation and decision-making. After every single draw, the conditionals of the parameters $(\beta \mid \mathbf{Y}$, for example) were updated. 10,000 identically and independently distributed samples were obtained. Figure 2 provides evidence of the properties of probabilistic inference of posterior parameters by clearly depicting crisis and non-crisis phases in the time series. By using thresholds

$\left(\theta_{\text {linf }}, \theta_{\text {med } 1}, \theta_{\text {med } 2}\right.$ and $\left.\theta_{\text {sup }}\right)$ the model can clearly delimit the two states in the time series. Detecting financial crises while they develop is essential, as contagion rapidly propagates liquidity shocks across interconnected financial markets. The consequences of such phenomena are manifested by the 2007-08 financial crisis, which had a devastating effect on several economies, triggering a prolonged and painful recession.

The model can predict the moment a liquidity crisis is about to strike with a high probability, and it is able to measure the persistence of crisis and noncrisis periods. The results fully support the fact that the interbank money market was in financial distress at least eight times for the analysed period including the financial crisis and the Eurozone crisis that followed. Compared to the approach of Kaminsky and Reinhart (2000) for example, who use the three standard deviations above the mean to classify the observations into crisis and non-crisis periods, this analysis uses a much more realistic approach to gauge the turbulent times for the analysed period. The mean 
and its standard deviation can only be representative of the data if it is normally distributed. However, as it has been demonstrated many times in the literature, asset prices and economic and financial rates are characterised by extreme lows and highs in times of financial or economic distress and therefore in such circumstances one cannot use standard econometric models to investigate market fundamentals. Our forecasting model yields superior posterior estimates compared to the estimates of the random walk model. Thus, the presented model is a first step and has the potential to benefit policy makers and institutional players alike. Bayesian inference proves to be very effective in estimating crisis and non-crisis regimes in short-term financial series. The main significance of the findings is that they can be used as a basis to develop an early warning system to detect liquidity shocks within the interbank market.

\section{Appendix A. Probability distribution and Bayesian learning in the crisis/non-crisis scenario}

Assume a normally distributed stochastic process with $\Theta$ being the set of parameters to be inferred, as follows:

$$
Y \sim N(\Theta)
$$

and

$$
\Theta=h\left(\theta, X^{1}, X^{2}, \ldots, X^{T}\right)
$$

where $X^{1}, X^{2}, \ldots, X^{T}$ are iterations of the coefficients to be inferred.

Let $\theta$ be the event that the times series of interest is in a state of financial crisis. Values of $\theta$ fall between 0 and 1. The likelihood function comprises

all the information fed by the sample and is determined by the equation

$$
p(Y \mid \theta)=\theta^{Y}(1-\theta)^{n-Y}
$$

with $Y=0,1, \ldots, n$. The evidence is determined by: 


$$
p(Y)=\int p(Y \mid \theta) p(\theta \mid Y) d \theta
$$

Therefore, the posterior distribution can be written as follows:

$$
p(\theta \mid Y)=\frac{p(Y \mid \theta) p(\theta)}{p(Y)}
$$

In other words, it now follows from Bayes' theorem that:

$$
p(\text { crisis } \mid \text { data })=\frac{p(\text { data } \mid \text { crisis }) p(\text { crisis })}{p(\text { data })}
$$

The probabilities related to various state changes are determined by:

$$
P_{k, l}=P\left(I_{t+1}=l \mid I_{t}=k\right)
$$

where $k, l \in 0,1, t=0,1, \ldots, T$. The equation tells us that the probability that the state variable $I$, for example, is in crisis in period $t+1$ given that $I$ was equal to $k$ in the previous period. Consequently, the model has four transition probabilities, which can be written in a matrix form, as follows:

$$
P=\left(\begin{array}{ll}
P_{00} & P_{01} \\
P_{10} & P_{11}
\end{array}\right)
$$

The conditional probability distribution of the data given crisis and non-crisis intervals, that is, the probability distribution of the data when the indicator variable is known to take the value of 0 or 1 , are defined as follows:

$$
\begin{gathered}
Y_{t} \mid I_{t}=0 \sim N\left(0, \sigma_{0, t}^{2}\right) \\
Y_{t} \mid I_{t}=1 \sim N\left(\rho Y_{t-1}, \sigma_{1, t}^{2}\right)
\end{gathered}
$$


The full conditional distribution is the distribution of the coefficients dependent on the known information and all the other coefficients (Lunn et al., 2013). In the non-crisis state $(I=0)$ the dependent variable follows a standard normal distribution with constant variance, whereas in the crisis state $(I=1)$ the variance changes persistently and increases with time. Furthermore, it is assumed that in crisis time, the dependent variable follows an autoregressive process of order 1 with first-order autoregressive coefficient $\rho$.

\section{Appendix B. Priors, likelihood estimation and Gibbs sampling}

The model is a typical hierarchical model (see Figure B.6). The parameters of the vector $\Theta$ follow prior distributions with the aim to handle uncertainties attached to predictions of crisis and non-crisis states. The prior distribution should contain all likely values of parameters which are to be estimated.

The latent state variable $\theta$ follows a categorical distribution, which is the generalisation of the Bernoulli distribution, and can take two values: 1 if there is a crisis state and 0 if there isn't:

$$
\theta \sim \operatorname{Cat}(P)
$$

The uniform (or noninformative or symmetric) distribution of the latent variable $\rho$ (which represents the autocorrelation coefficient), for example, is found within some boundaries as $a \rightarrow-\infty, b \rightarrow \infty:^{8}$

$$
\rho \sim \operatorname{Unif}(a, b)
$$

where $a$ and $b$ are the minimum and maximum values (boundaries) of the distribution, and the condition $b>a$ must be satisfied.

\footnotetext{
${ }^{8}$ The uninformative prior is characterised by a flat, vague and/or dispersed density, and so statistical inference is not influenced by information which is external to the data being analysed (Gelfand et al., 1990). Moreover, the distribution is not expected to be focused around the real or 'true' value, considering that information about the latent parameters comprised in the data will out-shadow any rational prior probability specification (Gelman et al., 2013).
} 
The transition probabilities $P_{11}$ and $P_{00}$ are assigned the Beta distribution with values in the range of $[0,1]$.

$$
\begin{aligned}
& P_{11} \sim \operatorname{Beta}(0.5,0.5) \\
& P_{00} \sim \operatorname{Beta}(0.5,0.5)
\end{aligned}
$$

To the remaining parameters of the model a uniform prior distribution is allocated over a range of values determined by thresholds, as follows:

The two lambdas describe the two volatility levels seen in the LIBOROIS spread fluctuations and both have two levels determined by thresholds depicted in Figure B.6.

$$
\begin{array}{r}
\rho \sim \operatorname{Unif}(0,1.5) \\
\lambda_{1} \sim \operatorname{Unif}\left(\theta_{\text {inf }}, \theta_{\text {med } 1}\right) \\
\lambda_{2} \sim \operatorname{Unif}\left(\theta_{\text {med } 2}, \theta_{\text {sup }}\right)
\end{array}
$$

The prior distributions for the four thresholds are defined as follows:

$$
\begin{array}{r}
\theta_{\text {low }} \sim \operatorname{Unif}(a, b) \\
\theta_{\text {med } 1} \sim \operatorname{Unif}\left(\theta_{\text {low }}, b\right) \\
\theta_{\text {med } 2} \sim \operatorname{Unif}\left(\theta_{\text {med } 1}, b\right) \\
\theta_{\text {sup }} \sim \operatorname{Unif}\left(\theta_{\text {med } 2}, b\right)
\end{array}
$$

For example, the threshold parameter $\theta_{\text {low }}$ has a prior which is uniformly distributed with the lower and upper limit $a$ and $b$ as the hyperparameters, whereas the threshold parameter $\theta_{\text {sup }}$ has a prior which is uniformly distributed between $\theta_{\text {med }_{2}}$ (representing the upper medium threshold) and $b$ (which is the upper level of the LIBOR-OIS spread observations).

Considering the crisis and non-crisis problem, the values of the hyperparameters $a$ and $b$ must be determined. $a$ can be set to the lowest value of all observations (the minimum and maximum values for the LIBOR-OIS series is 1.93 and 364.43, correspondingly), however for simplicity it is set to 0 , while the value of $b$ is set to 370 . The random variables are thus equally likely to take any values between $a$ and $b$. The probability of being in crisis or tranquil state is $50 \%$. 


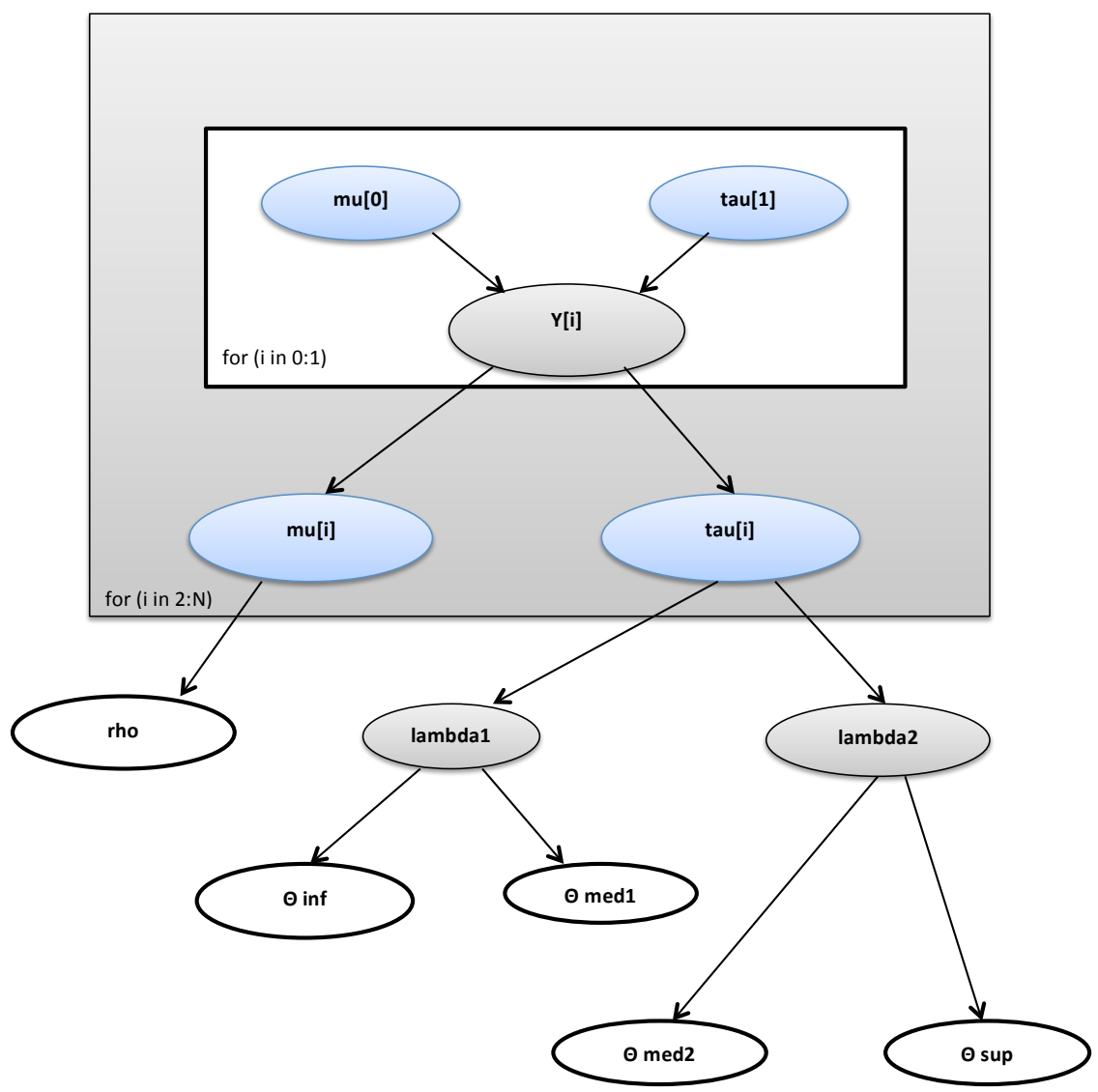

Figure B.6: Hierarchical structure of the two-state Markov regime switching model predicting crisis and non-crisis states. There are four thresholds, which show the escalation of liquidity risk within the interbank market. 
The Gibbs sampler proposed by Geman and Geman (1984) generates and predicts an array of forecasts from the joint density which then classifies the data into crisis and non-crisis periods. The algorithm begins with arbitrary initial values (for the elements of the parameter vector $\Theta$ ) set by the system itself. Based on the fact that the $Y_{t}$ process is explosive due to varying dynamics of the data, the crisis state is modelled as an autoregressive process. In the model specification, the values of $\rho$ are set to have a lower boundary of zero and upper boundary of 1.5. The non-crisis state is described by a Gaussian white noise process and the noise terms $\epsilon_{t}$ can take values between 0 and 370 (these values are the minimum and maximum values in the actual dataset, as explained earlier).

Posterior distribution of the parameters is obtained by combining the prior distributions with the likelihood function. The Gibbs sampler uses the conditional distributions to estimate the joint and marginal distributions. To obtain a sample from the posterior distribution, $J$ number of iterations are run. First, the state variable is sampled, such as $P(\theta \mid Y)$, by implementing state- and various threshold identifying restrictions (Martínez-Beneito et al., 2008) outlined from Equation B.8 to B.11:

1. The system defines initial values for the coefficients to be estimated;

2. Sample $X_{1}^{1}$ from $f\left(X_{1} \mid X_{1}^{0}, \ldots, X_{k}^{0}\right)$;

3. Sample $X_{2}^{1}$ from $f\left(X_{2} \mid X_{1}^{1}, X_{1}^{1}, \ldots, X_{k}^{0}\right)$;

4. Sample $X_{3}^{1}$ from $f\left(X_{3} \mid X_{1}^{1}, X_{2}^{1}, X_{4}^{0}, \ldots, X_{k}^{0}\right)$;

$\cdots$

n. To end one iteration, draw $X_{k}^{1}$ from $f\left(X_{k} \mid X_{1}^{1}, X_{2}^{1}, X_{4}^{0}, \ldots, X_{k}^{0}\right)$. The above process should be repeated $J$ times until convergence is achieved (Geman and Geman, 1984). Next, the transition parameters and the remaining parameters which are conditional on both the state parameter and data are sampled using the above steps.

1. Sample the remaining coefficients of the model using the steps from $1 \rightarrow n$;

2. Monitor convergence by plotting the posterior estimates.

\section{References}

Akaike, H. (1969). Fitting autoregressive models for prediction. Annals of the institute of Statistical Mathematics, 21(1):243-247. 
Ang, A. and Timmermann, A. (2011). Regime changes and financial markets. Technical report, National Bureau of Economic Research.

Baba, N., Packer, F., and Nagano, T. (2008). The spillover of money market turbulence to FX swap and cross-currency swap markets. BIS Quarterly Review, 3:73-86.

Barrell, R., Davis, E. P., Karim, D., and Liadze, I. (2010). Bank regulation, property prices and early warning systems for banking crises in OECD countries. Journal of Banking \& Finance, 34(9):2255-2264.

Bauer, C., Herz, B., and Karb, V. (2007). Are twin currency and debt crises special? Journal of Financial Stability, 3(1):59-84.

Berg, A., Borensztein, E., and Pattillo, C. (2005). Assessing early warning systems: how have they worked in practice? IMF staff papers, pages $462-502$.

Brunnermeier, M. (2009). Deciphering the liquidity and credit crunch 20072008. Journal of Economic Perspectives, 23:77-100.

Brunnermeier, M. K. and Oehmke, M. (2012). Bubbles, financial crises, and systemic risk. Technical report, National Bureau of Economic Research.

Bussiere, M. and Fratzscher, M. (2006). Towards a new early warning system of financial crises. Journal of International Money and Finance, 25(6):953 $-973$.

Caprio, G., Klingebiel, D., Laeven, L., and Noguera, G. (2012). Banking crises database. In Honohan, P. and Laeven, L., editors, Systemic Financial Crises: Containment and Resolution. Cambridge University Press.

Carpenter, S. B., Demiralp, S., and Senyuz, Z. (2016). Volatility in the federal funds market and money market spreads during the financial crisis. Journal of Financial Stability, 25:225-233.

Dahlquist, M. and Gray, S. F. (2000). Regime-switching and interest rates in the European monetary system. Journal of International Economics, 50(2):399-419. 
Daníelsson, J. and Saltoğlu, B. (2003). Anatomy of a market crash: A market microstructure analysis of the Turkish overnight liquidity crisis. In EFA 2003 Annual Conference Paper.

Davis, E. P. and Karim, D. (2008). Comparing early warning systems for banking crises. Journal of Financial Stability, 4(2):89 - 120.

Demirgüç-Kunt, A. and Detragiache, E. (1998). The determinants of banking crises in developing and developed countries. IMF Staff Papers, 45(1).

Demirgüç-Kunt, A. and Detragiache, E. (2005). Cross-country empirical studies of systemic bank distress: A survey. Technical Report WP/05/96, IMF.

Economist (2013). Crash course. Available: http://www.economist.com/news/schoolsbrief/

21584534-effects-financial-crisis-are-still-being-felt-five-years-article.

Flegal, J. M., Haran, M., and Jones, G. L. (2008). Markov chain Monte Carlo: Can we trust the third significant figure? Statistical Science, pages 250-260.

Frank, N., González-Hermosillo, B., and Hesse, H. (2008). Transmission of liquidity shocks: Evidence from the 2007 subprime crisis. International Monetary Fund Working Paper, 08(200).

Gelfand, A. E., Hills, S. E., Racine-Poon, A., and Smith, A. F. (1990). Illustration of Bayesian inference in normal data models using Gibbs sampling. Journal of the American Statistical Association, 85(412):972-985.

Gelman, A., Carlin, J., Stern, H., and Rubin, D. (2013). Bayesian Data Analysis. Taylor \& Francis, 3rd edition.

Gelman, A. and Rubin, D. B. (1992). Inference from iterative simulation using multiple sequences. Statistical Science, pages 457-472.

Geman, S. and Geman, D. (1984). Stochastic relaxation, Gibbs distributions, and the Bayesian restoration of images. IEEE Transactions on Pattern Analysis and Machine Intelligence, PAMI-6(6):721-741. 
Goldstein, M., Kaminsky, G. L., and Reinhart, C. M. (2000). Assessing financial vulnerability: an early warning system for emerging markets. Peterson Institute.

Gorton, G. and Metrick, A. (2012). Securitized banking and the run on repo. Journal of Financial Economics, 104(3):425 - 451.

Guo, F., Chen, C. R., and Huang, Y. S. (2011). Markets contagion during financial crisis: A regime-switching approach. International Review of Economics \&f Finance, 20(1):95 - 109.

Hartmann, P., Manna, M., and Manzanares, A. (2001). The microstructure of the euro money market. Journal of International Money and Finance, 20(6):895-948.

Hui, C.-H., Genberg, H., and Chung, T.-K. (2011). Funding liquidity risk and deviations from interest-rate parity during the Financial Crisis of 20072009. International Journal of Finance 8 Economics, 16(4):307-323.

Kaminsky, G., Lizondo, S., and Reinhart, C. M. (1998). Leading indicators of currency crises. Staff Papers - International Monetary Fund, pages 1-48.

Kaminsky, G. L. and Reinhart, C. M. (1999). The twin crises: the causes of banking and balance-of-payments problems. American Economic Review, pages 473-500.

Kaminsky, G. L. and Reinhart, C. M. (2000). On crises, contagion, and confusion. Journal of International Economics, 51(1):145 - 168.

Kim, C.-J. and Nelson, C. R. (1999). State-Space Models with Regime Switching: Classical and Gibbs-Sampling Approaches with Applications, volume 1 of MIT Press Books. The MIT Press.

Laeven, L. and Valencia, F. (2012). Systemic banking crises database: An update. Working Paper 12/163, IMF.

Lang, M. and Schmidt, P. G. (2016). The early warnings of banking crises: Interaction of broad liquidity and demand deposits. Journal of International Money and Finance, 61:1-29. 
Lunn, D., Jackson, C., Best, N., Thomas, A., and Spiegelhalter, D. (2013). The BUGS book: A practical introduction to Bayesian analysis. FL: CRC press.

Martínez-Beneito, M. A., Conesa, D., López-Quílez, A., and López-Maside, A. (2008). Bayesian Markov switching models for the early detection of influenza epidemics. Statistics in Medicine, 27(22):4455-4468.

McAndrews, J., Sarkar, A., and Wang, Z. (2008). The effect of the term auction facility on the London inter-bank offered rate. Federal Reserve Bank of New York. Staff Report.

Min, H.-G. and Hwang, Y.-S. (2012). Dynamic correlation analysis of US financial crisis and contagion: evidence from four OECD countries. Applied Financial Economics, 22(24):2063-2074.

Mistrulli, P. E. (2011). Assessing financial contagion in the interbank market: Maximum entropy versus observed interbank lending patterns. Journal of Banking 85 Finance, 35(5):1114 - 1127.

Schwarz, K. (2014). Mind the gap: Disentangling credit and liquidity in risk spreads. Available at: http://dx.doi.org/10.2139/ssrn.1486240.

Spiegelhalter, D., Best, N., Carlin, B., and Linde, A. (2002). Bayesian measures of model complexity and fit. Journal of the Royal Statistical Society, 64:583-639.

Thornton, D. L. (2009). What the LIBOR-OIS spread says. Available: https://research.stlouisfed.org/publications/es/09/ES0924.pdf.

Upper, C. (2011). Simulation methods to assess the danger of contagion in interbank markets. Journal of Financial Stability, 7(3):111-125.

Zhou, W. and He, J. (2012). A novel financial risk contagion model based on the MGARCH process and its parameter estimation. African Journal of Business Management, 6(19):6014-6018. 